\title{
Determinação dos parâmetros $\alpha$ e b da função de Cobb - Douglas, através do método dos mínimos quadrados
}

\author{
João Socorro Pinheiro Ferreira Nágela Rafaela Bastos Silva \\ Colegiado de Licenciatura em Matemática \\ Universidade Federal do Amapá, UNIFAP \\ E-mail: joaoferreira@unifap.br \\ 68.903-419 - Macapá - Amapá
}

\begin{abstract}
RESUMO
Este texto é resultado de projeto de pesquisa dos acadêmicos supracitados, sob orientação do Professor João Ferreira, cuja temática é a matemática aplicada. Tendo como objetivo principal a utilização de um método numéricos para a obtenção das constantes da solução de problemas científicos, mais especificamente a solução da EDP modeladora do problema de Cobb-Douglas - dois americanos que na década de vinte do século XX, propuseram um modelo matemático para a previsão da economia americana, em um período de vinte e quatro anos - utilizando dados oficiais dos boletins econômicos americanos. Pesquisou-se em várias obras do gênero - sendo que algumas apresentam a EDP de CobbDougras e a sua solução algébrica - no caso uma função de duas variáveis reais, mas nenhuma mostra como se obter os parâmetros $\alpha$ e b. Sendo assim, neste trabalho, a partir dos dados fornecidos por [3], linearizou-se a função solução e com auxílio da planilha Excel, obteve-se os parâmetros desconhecidos. Ao final do trabalho, são discutidos os resultados da solução da EDP de CobbDouglas, com a simulação de alguns valores para medir o erro existente.
\end{abstract}

Palavras-chave: Matemática Aplicada, Modelagem, EDP, Iniciação Científica, Parâmetros.

\section{INTRODUÇÃO}

Em 1928, Charlles Cobb e Paul Douglas publicaram um estudo no qual modelava o desempenho da economia norte americana durante o período de 1899 a 1922. Eles adotaram uma visão simplificada na qual a produção era determinada pela quantidade de capital investido e mão de obra empregada. Apesar de existirem muitos outros fatores afetando o desempenho da economia, o modelo mostrou-se bastante preciso. A função potência encontrada para modelar a produção é mostrada na equação (1).

$$
P(L, K)=b L^{\alpha} K^{1-\alpha}
$$

Sendo $P$ é a produção total, $L$, a quantidade de mão de obra e $K$ a quantidade de capital investido. A Equação 1 é a solução da EDP, encontrada na obra [3], conforme Equação 2.

$$
\frac{\partial P}{\partial L} \frac{d L}{d t}+\frac{\partial P}{\partial K} \frac{d K}{d t}=P
$$

Desse modo, para atingir os propósitos deste trabalho, primeiramente linearizou-se a Equação 1 e para determinar os parâmetros $\boldsymbol{\alpha}$ e $\mathbf{b}$, através do método dos mínimos quadrados de Gauss.

A linearização da Equação 1, é a seguinte (EQUAÇÃO 3):

$$
\operatorname{Ln}\left(\frac{P}{K}\right)=\operatorname{Ln}(b)+\alpha \operatorname{Ln}\left(\frac{L}{K}\right)
$$

E pode ser comparada a Equação 4:

$$
Y=A+B x
$$

Sendo que seus coeficientes são:

$$
A=\operatorname{Ln}(b)
$$

$\mathrm{e}$

$$
B=\alpha
$$

Os interceptos A e B (EQUAÇÃO 4) serão calculados a partir do sistema mostrado em [1] (EQUAÇÃO 8). Posteriormente, substituiremos nas equações (5) e (6). Lembramos que a da equação (5) pode-se tirar o parâmetro $b$, que é um dos propósitos deste trabalho, e da equação (6), temos imediatamente o parâmetro alfa $(\alpha)$. O Valor de $b$ é obtido por:

$$
b=e^{A}
$$

\section{METODOLOGIA}


A obtenção da Equação 1 encontra-se em [3]. Para determinar os parâmetros mencionados, aplicou-se o método dos mínimos quadrados, a partir das demonstrações do método abordado por [1] e [2]. Sendo que em seus estudos, os autores deste artigo, primeiramente efetuaram a linearização da função exponencial e a tabulação dos dados, conforme mostrados na Tabela 1, que está em [3, p. 78] .

Para determinar os interceptos A e B, utilizou - se o sistema linear, mostrado na Equação 8. Os somatórios da última linha da Tabela 1, foram substituídos na Equação 3:

$$
\left\{\begin{array}{c}
n A+B \sum_{i=1}^{n} x_{i}=\sum_{i=1}^{n} y_{i} \\
A \sum_{i=1}^{n} x_{i}+B \sum_{i=1}^{n} x_{i}^{2}=\sum_{i=1}^{n} x_{i} y_{i}
\end{array}\right.
$$

A solução do sistema é A $=0,006902618755$ e $\mathrm{B}=0,7442384666$.

\section{RESULTADOS ALCANÇADOS}

Substituindo - se os valores de A e B nas Equações 5 e 6 , determina - se $\boldsymbol{\alpha}=\mathbf{0 , 7 4 4 2 3 8 4 6 6}$ e b = 1,006926496, que arredondando - se para duas casas decimais, tem-se os resultados existentes em [3].

Substituindo-se o valor de $\boldsymbol{\alpha}$ e $\mathbf{b}$, na Equação 1, tem-se:

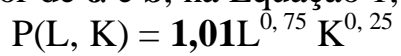

A Equação 9, representa a modelagem da economia americana no período citado na durante todo este trabalho.

Após atingir os propósitos deste trabalho, verificaremos a seguir se os mesmos irão produzir os resultados existentes na referência [3]. Para isso, calculou-se a produção nos anos de 1910 e 1920, a partir da mão de obra e do capital investidos naquela época e obtiveram-se os valores aproximados a saber:

$$
\begin{gathered}
1910 \quad \Rightarrow \quad \mathrm{P}(147,208)=1,01 \times(147)^{0,75} \times(208)^{0,25}=161,9 \\
1920 \quad \Rightarrow \quad \mathrm{P}(194,407)=\mathbf{1 , 0 1} \times(194)^{0,75} \times(407)^{0,25}=235,8
\end{gathered}
$$

que estão muito próximos dos valores dados de acordo com [3].

Com isso, o modelo proposto por Cobb-Douglas mostrou-se satisfatório para os dados da economia americana durante os 24 anos estudados, conforme os resultados obtidos acima.

\section{REFERÊNCIAS}

[1] BARROSO, L. C.; BARROSO, M. M. A.; CAMPOS FILHO, F. F.; CARVALHO, M. L. B.; MAIA, M. L. Cálculo numérico: com aplicações. 2. ed. São Paulo: Editora Harbra, 1987.

[2] BURIAN, Reinaldo; LIMA, Antonio Carlos de; HETEM JUNIOR, Annibal. Cálculo numérico. Reimpr. Rio de Janeiro: 2011. (Fundamentos de Informática).

[3] STEWART, James. Calculo: volume II. São Paulo: Cengage Learning, 2011. 